\title{
L'arrêt maladie, motif de licenciement en droit chypriote
}

\section{Matina Yannakourou}

\section{(2) OpenEdition \\ 1 Journals}

\section{Édition électronique}

URL : https://journals.openedition.org/rdctss/1644

DOI : $10.4000 /$ rdctss. 1644

ISSN : 2262-9815

Éditeur

Centre de droit comparé du travail et de la sécurité sociale

\section{Édition imprimée}

Date de publication : 1 avril 2019

Pagination : 74-87

ISSN : 2117-4350

\section{Référence électronique}

Matina Yannakourou, «L'arrêt maladie, motif de licenciement en droit chypriote », Revue de droit comparé du travail et de la sécurité sociale [En ligne], 1 | 2019, mis en ligne le 01 novembre 2021, consulté le 13 novembre 2021. URL : http://journals.openedition.org/rdctss/1644 ; DOI : https:// doi.org/10.4000/rdctss. 1644

\section{(c) (i) $\odot$}

Revue de droit comparé du travail et de la sécurité sociale est mise à disposition selon les termes de la Licence Creative Commons Attribution - Pas d'Utilisation Commerciale - Pas de Modification 4.0 International. 


\title{
L'ARRÊT MALADIE, MOTIF DE LICENCIEMENT EN DROIT CHYPRIOTE
}

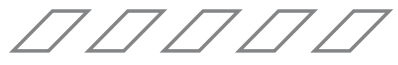

\section{RÉSUMÉ}

Une des conséquences de la crise financière chypriote de 2012-2013 sur les relations de travail fut l'augmentation du nombre des licenciements des salariés longuement absents pour cause de maladie dès leur retour au travail. Ce phénomène a suscité l'intervention du législateur en 2016. L'étude analyse le contexte et le contenu de la réforme légale; elle s'interroge aussi sur l'articulation de la nouvelle norme avec la norme préexistante qui autorisait le licenciement pour insuffisance professionnelle, à la lumière de la Charte sociale européenne révisée et de la jurisprudence nationale.

MOTS CLÉS : Maladie, licenciement, handicap, discrimination, inaptitude professionnelle, Chypre.

\begin{abstract}
One of the consequences of the Cypriot financial crisis of 2012-2013 on labour relations was the increase in the number of dismissals of employees who had been absent for a long time because of illness, as soon as they returned to work. This phenomenon provoked the intervention of the legislator in 2016. The study analyses the context and the content of the legal reform; it also examines the articulation of the new rule with the existing rule that authorises dismissal for professional incompetence, in the light of the revised European Social Charter and national case law.
\end{abstract}

KEYWORDS : Illness, Dismissal, ilnvalidity, Discrimination, Unfitness for Work, Cyprus. 


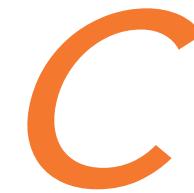

et article examine les conditions de validité d'un licenciement ayant pour motif les arrêts de travail du salarié pour cause de maladie, à travers l'étude du droit chypriote. La première partie offre un tour d'horizon du régime juridique de la rupture du contrat de travail et le rôle du juge dans son contrôle de légalité (I); la seconde analyse le contexte et le contenu de la réforme légale en matière de licenciement pour arrêt maladie ayant eu lieu en 2016 (II); enfin, la dernière partie s'interroge sur l'articulation de la nouvelle norme avec la norme qui consacre la validité du licenciement pour insuffisance professionnelle, à la lumière de la Charte sociale européenne révisée et de la jurisprudence nationale (III).

\section{I - LE RÉGIME JURIDIQUE DE LA RUPTURE DU CONTRAT DE TRAVAIL}

\section{A - LE CADRE GÉNÉRAL ET LA NATURE DU CONTRÔLE JUDICIAIRE}

La cessation du contrat de travail à durée indéterminée à l'initiative de l'employeur (licenciement) en Chypre est régie par la loi relative à la cessation d'emploi (Loi 24/1967); sa dernière modification a eu lieu en juillet 2016 et portait sur le traitement juridique de l'arrêt maladie en tant que motif de licenciement.

Inspirée par la Recommandation n 119 de 1963 de l'OIT $^{1}$ et l'article 24 de la Charte sociale européenne révisée, la loi 24/67 est structurée autour de deux piliers : d'une part l'interdiction de licencier sans cause réelle et sérieuse, de l'autre l'obligation d'indemniser le salarié seulement lorsque le licenciement s'avère non fondé sur un motif valable. En droit chypriote, le licenciement du salarié n'est permis que pour l'un des motifs énumérés de façon exhaustive dans la loi, faute de quoi le salarié licencié a droit à une indemnité compensatoire en fonction de son ancienneté dans l'entreprise et à sa réintégration sous certaines conditions.

La loi définit les motifs valables de la cessation d'emploi, les obligations qui incombent à l'employeur lorsqu'il met fin au contrat de travail - de manière licite ou illicite - et les droits du salarié déclaré excédentaire. Les vingt-six (26) premières semaines après le recrutement constituent une période d'essai pendant laquelle chacune des deux parties peut résilier le contrat de travail sans préavis ni indemnités. Tout travailleur licencié en tant que personnel excédentaire, n'ayant pas atteint l'âge de la retraite et ayant accompli un service d'au moins cent quatre (104) semaines chez le même employeur, a droit à une indemnité spéciale versée par la Caisse du personnel excédentaire.

1 Remplacée par la Recommandation n 166 de 1982 sur le licenciement. 
La loi $n^{\circ} 24$ de 1967 est la pierre angulaire de la législation chypriote de travail et son

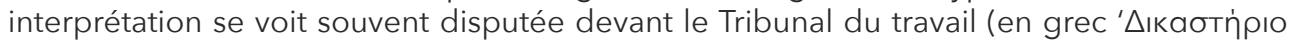

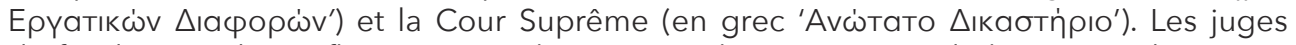
du fond, saisis de conflits entre employeurs et salariés émanant de la rupture du contrat de travail, recherchent une solution juste et équitable applicable au cas par cas. Pour déterminer la solution équitable on se réfère au comportement hypothétique de l'homme prudent moyen ( "l'homme raisonnable») et sa perception des choses². Pour se rendre compte de ce trait de spécificité de la fonction du juge en droit du travail, il faut remonter aux origines du droit chypriote.

Le corps principal des lois du travail chypriotes a été développé aux lendemains de période de l'occupation britannique (1878-1960), avec la proclamation de Chypre comme République indépendante en 1960. A cette époque, le système juridique du pays avait déjà reçu et retenu des influences culturelles diverses : d'une part la longue tradition du Common law anglais et des règles d'équité ${ }^{3}$, qui avaient infiltré la majorité des branches du droit civil, y compris celle de la procédure civile; d'autre part les empreintes du droit romain, byzantin et ottoman pour ce qui est du foncier. Enfin, après 1960, la pénétration des droits grec et français dans le domaine du droit public chypriote, notamment du droit administratif4.

Le droit régissant en particulier le contrat individuel de travail porte la forte empreinte de la doctrine du Common law anglais et de la règle du précédent; qui oblige à appliquer les décisions juridictionnelles, à savoir l'accumulation des décisions de principe et en particulier les motifs et les considérations de droit (ce qu'on appelle la 'ratio decidendi' de la décision), à des litiges postérieurs ${ }^{5}$. Outre ses origines du Common law, le droit du travail revêt à la fois le caractère d'un droit autochtone, en raison du degré élevé de pénétration des sources juridiques internationales - telles que la Convention Européenne des Droits de l'Homme, les conventions internationales de l'OIT ainsi que la Charte Sociale Européenne - dans le droit statutaire, au stade même de son élaboration. Cette branche juridique reflète alors l'effort d'instituer un droit postcolonial ayant les caractéristiques d'un système juridique mixte', à la fois d'un droit coutumier ou jurisprudentiel ('Common law') et d'un droit écrit ('Statute law'). La législation du travail est restée minimale jusqu'à l'adhésion de Chypre à I'Union Européenne (2004); à partir de là elle s'est enrichie d'un grand nombre de lois adoptées en vue d'une harmonisation du droit interne avec l'acquis social de l'Union. Le mélange de traditions et de cultures juridiques multiples a contribué à la formation d'un

2 G.M. Pikis, G.M., Aspects essentiels du droit chypriote (en grec), Ant. N. Sakkoulas, Athènes, 2003, p. 36.

3 La notion d'équité (en anglais 'equity') renvoie à l'ensemble des règles de clémence édictées au Royaume-Uni depuis le XVème siècle par la Cour de la Chancellerie à laquelle avaient recours les parties au procès qui cherchaient à modérer les effets d'une application stricte des règles du droit commun.

4 S. Symeonides, "The Mixed Legal System of the Republic of Cyprus", Tulane Law Review, 2003; $n^{\circ} 78$, p. 441.

5 En revanche, dans des systèmes relevant du droit continental les décisions prononcées par une juridiction le sont pour des cas concrets: la "jurisprudence » est alors à entendre comme l'ensemble de ces décisions concrètes, leur portée normative étant limitée par l'autorité relative de la chose jugée.

6 N. Hatzimichael, N., "Cyprus as a Mixed Legal System", Journal of Civil Law Studies, n 6, vol. 1, 2013, p. 38. 
droit hybride, dont la décodification est loin d'être aisée pour les chercheurs étrangers, voire pour les comparatistes.

L'une des caractéristiques du droit du travail chypriote est qu'il incarne la logique de la mise en balance des intérêts de l'employeur et du travailleur, afin de rechercher une solution juste et équitable à tout conflit individuel de travail ${ }^{7}$. Cette logique est le résultat de l'influence exercée par la pratique judiciaire d'équité sur le droit chypriote, à travers l'application des principes du droit des contrats, une branche du droit privé qui demeure entièrement ancrée dans la doctrine du Common law anglais. Les principes du droit des contrats, ayant leur racine dans le Common law, s'appliquent au contrat de travail dans la mesure où ils ne sont pas en concurrence avec les normes étatiques en la matière. Un de ces principes est celui du comportement raisonnable ('the principle of reasonableness').

Le principe du comportement raisonnable est un principe général du droit du travail chypriote qui guide le raisonnement juridique du juge sur le bien-fondé du licenciement. En effet, le juge de fond confronte l'attitude de l'employeur en question avec l'attitude d'un employeur moyen qui se montrerait raisonnable et prudent vis-à-vis de ses salariés. Par exemple, lorsque le juge est saisi de la question de licéité d'un licenciement pour mauvaise conduite du salarié, il examine si un employeur normalement prudent, dans les circonstances de l'espèce, pourrait raisonnablement conclure que le comportement du salarié était si déplorable qu'il pourrait justifier la rupture du contrat de travail. De la même façon, l'employeur doit décider, en se mettant à la place d'un employeur raisonnable dans les circonstances précises, s'il va mettre un terme à l'emploi de son salarié pour inaptitude professionnelle, sachant que la charge de la preuve du motif du licenciement pèse sur lui ${ }^{8}$. Le critère pertinent à cet effet, évalué par la Cour Suprême dans son jugement, est si l'employeur a conclu, comme un employeur raisonnable au vu des circonstances, qu'il fallait procéder à une cessation d'emploi sur la base des données dont il dispose?. La charge de la preuve incombe à l'employeur mais l'affaire sera tranchée sur une évaluation des probabilités qui caractérise le système de la preuve en droit chypriote ${ }^{10}$. Par ailleurs, c'est cette approche qui diversifie le droit chypriote, en tant que droit relevant du système du Common law anglais, des droits du système continental basés sur le droit romain. Dans ces derniers le juge recherche s'il y a des droits en vertu de la loi que le tribunal doit reconnaître et satisfaire en appliquant les règles de droit au cas d'espèce ${ }^{11}$. En revanche, le juge chypriote adopte la méthode de la mise en balance des intérêts de l'employeur et

7 S. Yannakourou, S., Droit du Travail Chypriote (en grec), Nomiki Vivliothiki, Athènes, 2016, p. 40.

8 P. Polyviou, P., Le Droit du Travail à Chypre. Théorie et Pratique (en grec), Chryssafinis et Polyviou ed., Nicosie, 2017, p. 305.

9 Comme l'a expliqué Lord Denning dans l'arrêt anglais British Leyland (UK) Ltd v. Swift (1981) 1 RLR 91, 93, ayant établi le précédent qui a été par la suite adopté en droit chypriote dans l'arrêt-phare Kakofengitou v. Cyprus Airways Ltd (2005) 1 Arrêts de la Cour Suprême 603 : «The correct test is this: Was it reasonable for the employers to dismiss him? If no reasonable employer would have dismissed him, then the dismissal was unfair. But if a reasonable employer might reasonably have dismissed him, then the dismissal was fair. It must be remembered in all these cases there is a band of reasonableness, within which one employer might reasonably take one view, another quite reasonably take a different view».

10 Antonis Staphylidis v. Nemesis Entreprise Publique Contractante Ltd, Tribunal du Travail Application $\mathrm{n}^{\circ} 101 / 2011$, arrêt du 5.5.2015.

11 G.M. Pikis, préc., p. 43. 
du travailleur, aux fins de la recherche de la solution pertinente et équitable à appliquer au cas par cas ${ }^{12}$.

\section{B - LE LICENCIEMENT POUR CAUSE JUSTE}

Comme déjà évoqué, le salarié peut être valablement licencié pour des motifs limitativement énumérés par la loi ${ }^{13}$, à savoir :

- le salarié n'exécute pas son travail de manière satisfaisante;

- le salarié est en sur effectif;

- la cessation d'emploi est due à un cas de force majeure, une guerre, une insurrection, une catastrophe ou une destruction de foyer en raison d'un incendie involontaire ou d'une négligence du salarié;

- le salarié a un contrat de travail à durée déterminée qui arrive à terme, sauf si la durée du contrat excède trente (30) mois, ce qui constitue une présomption de contrat de travail à durée indéterminée;

- le salarié a atteint l'âge normal de la retraite en vertu de la loi, de la coutume, de la convention collective, du contrat ou de la profession;

- le salarié est susceptible de licenciement sans préavis en raison de sa conduite. Dans ce dernier cas, l'employeur est exonéré de l'obligation de donner un délai de préavis et peut donc renvoyer le salarié immédiatement en raison de son comportement, indépendamment de ses performances.

La loi édicte que, notamment les événements suivants peuvent constituer un motif de licenciement sans préavis, en fonction des circonstances de l'espèce:

- faute grave commise par le salarié dans l'exercice de ses fonctions;

- commission d'une infraction pénale par le salarié dans l'exercice de ses fonctions sans le consentement exprès ou tacite de l'employeur ;

- conduite injurieuse du salarié au moment de l'exécution de ses fonctions;

- violation grave ou répétée des règlements du travail ou d'autres règles en matière d'emploi.

\section{C - LE LICENCIEMENT ILLÉGAL}

Lorsque la lettre de licenciement adressée au salarié ne comporte pas un motif de licenciement parmi ceux limitativement énumérés par la loi, le licenciement est sans cause réelle et sérieuse, à savoir illégal. Aux fins de la protection du salarié, tout licenciement est présumé illégal et il appartient à l'employeur de prouver qu'il est probablement advenu pour l'un des motifs prévus par la loi. En outre, la loi ${ }^{14}$ prescrit les motifs spéciaux qui rendent la rupture du contrat de travail illégale et qui sont les suivants :

- La qualité du salarié comme membre d'un syndicat ou d'un comité de santé et de sécurité de travail;

12 S. Yannakourou, préc., pp. 40-41.

13 Art. 5 de la Loi 24/67.

14 Article 6(2) de la Loi 24/67. 
- La participation du salarié à des activités syndicales en dehors des heures de travail ou dans le cadre de l'horaire du travail mais avec le consentement de l'employeur;

- L'état de grossesse ou de maternité;

- La prise d'un congé parental ou d'un congé pour des raisons de force majeure;

- La race, la couleur, le sexe, l'état matrimonial, la religion, les opinions politiques, l'origine nationale, l'origine sociale;

- Le dépôt d'une plainte ou la participation du salarié à une procédure civile ou pénale contre l'employeur.

\section{II - L'ABSENCE POUR CAUSE DE MALADIE COMME MOTIF DE LICENCIEMENT}

\section{A - LA PROBLÉMATIQUe GÉNÉRALE}

Le contrat de travail étant un contrat synallagmatique, une maladie temporaire ne devrait en principe susciter qu'une suspension de l'exécution de la prestation de travail de la part du salarié et une exonération réciproque de l'employeur de verser le salaire, faute de contrepartie de la part de son cocontractant. L'avènement d'une maladie ne saurait entraver la continuation du contrat de travail et le maintien en vigueur des obligations contractuelles. Mais que se passe-t-il lorsqu'on est en présence d'une maladie de longue durée ou d'absences fréquentes et répétées qui peuvent influer sur la capacité du travail et/ ou sur le bon fonctionnement de l'entreprise? Un tel fait pourrait-il - et à quelles conditions conduire l'employeur à envisager la rupture du contrat, non pas nécessairement du fait de l'état de santé lui-même, mais à cause de ses conséquences sur la disponibilité du salarié et sur l'exercice régulier de ses fonctions?

II n'est pas exclu qu'une loi pose des plafonds légaux au nombre de jours d'absence justifiée du travail pour cause de maladie, au-delà desquels la résiliation du contrat de travail par l'employeur est en principe autorisée. On voit aussi apparaître des variables de ce modèle qui optent pour une définition légale de l'arrêt maladie de courte durée dans le but de tracer explicitement la ligne de démarcation entre ce dernier et les absences au-delà de celle-ci ou les absences répétées ou intermittentes. En droit grec, par exemple, la loi conditionne la qualification et la durée d'une absence de maladie à court terme à l'ancienneté du salarié dans l'entreprise ${ }^{15}$.

L'incidence pratique d'une telle distinction est que l'absence pour maladie à court terme est considérée comme justifiée, alors que le traitement juridique de la maladie prolongée se fait au cas par cas selon les critères établis par la jurisprudence. Ces dispositions

15 Article 5 al. 3 de la loi 2112/1920. En vertu de cette loi est qualifiée de maladie à court terme : l'absence continue i) d'un (1) mois pour les salariés ayant une ancienneté de moins de quatre (4) ans; ii) de trois (3) mois pour les salariés comptant entre quatre (4) et dix (10) années de service chez le même employeur, iii) l'absence continue de quatre (4) mois pour ceux qui font preuve d'une ancienneté de dix (10) à quinze (15) ans et iv) l'absence continue de six (6) mois pour ceux qui sont au service du même employeur depuis plus de quinze (15) ans. 
s'appliquent à toute forme d'arrêt-maladie quelle que soit sa cause ${ }^{16}$. Le dépassement des limites maximales fixées par la loi n'entraîne pas en soi la cessation automatique du contrat de travail. Donc, l'arrêt de travail pendant une période qui dépasse la dite 'période de garantie d'emploi' n'équivaut pas à une démission tacite du salarié et n'entraîne pas automatiquement la résiliation du contrat de travail. II appartient au juge de déterminer, dans chaque cas particulier, en tenant compte des circonstances dans lesquelles s'est effectuée l'absence du salarié, si, indépendamment de l'intention de ce dernier de mettre fin au contrat, il peut être tiré de manière objective, conformément à la bonne foi contractuelle, qu'il s'agissait bel et bien d'une résiliation implicite du contrat ${ }^{17}$. Ainsi, en droit grec le licenciement du salarié pour arrêt maladie, donnant lieu à un pronostic négatif sur le bon déroulement de la relation de travail, est-il considéré comme ne dépassant pas les contours de l'abus de droit énoncés à lıarticle 281 du Code civil grec, à savoir les limites posées par la bonne foi, les bonnes mœurs ou le but social et économique du droit en cause $^{18}$.

\section{B - LE VIDE JURIDIQUE AVANT 2016}

Contrairement à l'analyse précitée, la législation chypriote ne contient aucune définition légale de la notion d'absence temporaire pour cause de maladie. De surcroit, elle ne fait aucune distinction entre maladie de courte durée e et maladie prolongée ou de longue durée.

L'état de maladie du salarié n'étant pas inclus dans la liste restrictive des motifs qui rendent la résiliation du contrat de travail illégale, la question s'est posée de savoir si l'absence pour cause de maladie pourrait entraîner - et à quelles conditions - un tel effet. La question est d'actualité car la récession économique ayant frappé le pays à partir de 2012-2013 a engendré de multiples licenciements des salariés longuement absents pour cause de maladie dès leur retour au travail. La réponse n'allait pas de soi; la lecture par combinaison des dispositifs du droit du travail et des dispositifs du droit de la sécurité sociale a révélé l'existence d'un vide juridique en la matière.

A l'époque, la loi chypriote en matière de rupture du contrat de travail ${ }^{19}$ interdisait à l'employeur de donner un préavis de licenciement à un salarié en arrêt maladie pendant les six (6) mois suivant le premier jour d'absence. L'hypothèse d'une absence au-delà des six mois n'était point visée par la disposition en cause. En même temps, la loi sur la sécurité sociale ${ }^{20}$ prévoit que le salarié peut recevoir des prestations de maladie pendant cent cinquante-six (156) jours supplémentaires (environ six mois), à condition que la maladie persiste et que

16 Arrêt de la Cour de Cassation (Areios Pagos) 542/2010, Epitheorissi Ergatikou Dikaiou (EErgD) 2010.1514 (en grec).

17 Articles 200 et 288 du Code Civil Grec et la jurisprudence y relative. Voir l'analyse de D. Zerdelis, Droit individuel du travail, ed. Sakkoulas, 2015, p. 1179.

18 D. Goulas, Les discriminations à l'encontre des salariés ayant des problèmes de santé mentale, EErgD, 2018, p. 513.

19 Article 9(6) de la Loi 24/67.

20 Article 31(3) de la Loi 59(I)/2010. 
le salarié ne soit pas déclaré en état d'incapacité professionnelle permanente. Au cours de cette période de couverture sociale supplémentaire de six mois, le salarié avait le droit de recevoir une indemnité de maladie, mais était exclu de la protection contre le licenciement offerte par la loi 24/67. Il y avait alors un vide juridique sur la question du licenciement pour arrêt de maladie prolongée, parce que les deux lois, - celle relative à la fin de la cessation du travail et celle relative à l'assurance sociale du travail -, n'étaient pas alignées. Toutefois, le salarié n'était pas totalement démuni de protection, car il bénéficiait de l'armature juridique établie par la loi 127(I)/2000 qui a harmonisé le droit chypriote avec la Directive 2000/78 en matière de la non-discrimination ${ }^{21}$.

\section{LE LICENCIEMENT POUR ARRÊT MALADIE COMME DISCRIMINATION}

Bien que l'assimilation pure et simple de la notion de maladie à celle de handicap soit exclue par la jurisprudence de la CJUE$^{22}$, il n'empêche que le licenciement ayant pour motif les absences du salarié pour cause de maladie liées à un handicap, peut être considéré comme une discrimination au sens de la directive 2000/78/EC. Cette directive a été transposée en droit chypriote par la loi 127(I)/2000.

La directive 2000/78 a pour objet d'établir un cadre général pour lutter, en ce qui concerne l'emploi et le travail, contre les discriminations fondées sur l'un des motifs visés à son article 2 paragraphe 2, sous b) i), au nombre desquels figure le handicap. Cette directive consacre le principe de l'égalité de traitement, entendu comme étant l'absence de toute discrimination directe ou indirecte, fondée sur un des motifs visés par celle-ci. Le principe de l'égalité de traitement s'applique, entre autres, aux conditions de licenciement. Selon la jurisprudence de la Cour, la notion de «handicap ", au sens de la directive 2000/78, doit être entendue comme visant une limitation des capacités, résultant notamment d'atteintes physiques, mentales ou psychiques durables, dont l'interaction avec diverses barrières peut faire obstacle à la pleine et effective participation de la personne concernée à la vie professionnelle sur la base de l'égalité avec les autres travailleurs ${ }^{23}$. Donc, pour entrer dans le champ d'application de la directive, la limitation de la capacité du salarié de travailler doit être qualifiée de handicap au sens de cette directive.

La Directive exige que l'employeur prenne des mesures appropriées d'aménagement raisonnable pour faciliter l'accès et la participation à l'emploi des personnes handicapées ${ }^{24}$. Le licenciement du salarié ne devrait pas être fondé sur les absences pour cause de maladie si ces absences sont dues au manquement de l'employeur d'adopter des mesures d'aménagement raisonnable ${ }^{25}$. L'employeur ne peut pas invoquer les absences du salarié

21 Directive 2000/78/CE du 27 novembre 2000, portant création d'un cadre général en faveur de l'égalité de traitement en matière d'emploi et de travail.

22 Arrêt de la CJUE du 11 juillet 2006, Chacón Navas, C13/05, EU:C:2006:456, point 44, Arrêt du 11 avril 2013, HK Danmark, C335/11 et C337/11, EU:C:2013:222, point 75.

23 Arrêt de la CJUE du 9 mars 2017, Milkova, C406/15, EU:C:2017:198, point 36 et jurisprudence citée.

24 Article 9 de la loi 127(I)/2000.

25 HK Danmark, préc., points 66-67. 
à son profit quand celles-ci résultent de son manquement à prendre des ajustements raisonnables pour permettre au salarié de satisfaire aux exigences de son travail. Un tel comportement constituerait une violation de la Directive 2000/78 et de la loi 127(I)/2000.

De surcroît, en vertu d'un arrêt important de la CJUE, ${ }^{26}$ est considérée comme contraire à la directive 2000/78 - et donc comme constitutif de discrimination fondée sur le handicap-, une réglementation nationale qui prévoit qu'un employeur peut licencier un travailleur pour le motif tiré d'absences intermittentes au travail, fussent-elles justifiées, dans la situation où ces absences sont la conséquence de maladies imputables au handicap dont est atteint ce travailleur. En l'occurrence, la disposition de la loi espagnole en cause énonçait qu'un contrat de travail peut être résilié pour le motif tiré d'absences intermittentes au travail, fussent-elles justifiées, qui représentent $20 \%$ des jours ouvrables au cours de deux mois consécutifs, lorsque le total des absences au cours des douze mois précédents atteint $5 \%$ des jours ouvrables ou $25 \%$ au cours de quatre mois non consécutifs sur une période de douze mois.

La lutte contre l'absentéisme au travail peut être considérée comme un objectif légitime, au sens de la directive 2000/78, dès lors qu'il s'agit d'une mesure relevant de la politique nationale de l'emploi ${ }^{27}$, pourvu que les exigences posées par le principe de proportionnalité soient respectées. II importe cependant de vérifier si les moyens mis en œuvre par la réglementation nationale pour réaliser cet objectif sont appropriés et n'excèdent pas ce qui est nécessaire pour atteindre celui-ci. Pour évaluer le caractère approprié des moyens mis en œuvre à cet égard, il faut vérifier a) si les données chiffrées regroupées sont effectivement conçues pour répondre à l'objectif consistant à lutter contre l'absentéisme au travail, sans couvrir des absences qui seraient simplement ponctuelles et sporadiques; b) les coûts directs et indirects que doivent supporter les entreprises du fait de l'absentéisme au travail; c) si la législation nationale, en prévoyant le droit de procéder au licenciement des travailleurs absents de manière intermittente au travail pour cause de maladie pendant un certain nombre de jours, a, vis-à-vis des employeurs, un effet incitatif à l'embauche et au maintien en fonction ${ }^{28}$.

Dans l'appréciation du caractère proportionné des moyens utilisés, il convient également de ne pas méconnaître le risque encouru par les personnes atteintes d'un handicap, lesquelles rencontrent en général davantage de difficultés que les travailleurs valides pour réintégrer le marché de l'emploi et ont des besoins spécifiques liés à la protection que requiert leur état ${ }^{29}$.

En résumé, le juge chypriote, saisi d'un litige relatif à un licenciement pour absences fréquentes et répétitives en raison d'une maladie liée à un handicap, devra interpréter le droit national conformément aux dispositions de la directive 2000/78 30 et à leur

26 Arrêt de la CJUE du 18 janvier 208, Ruiz Conejero, C-270/16, ECLI:EU:C:2018:17.

27 HK Danmark, préc., point 82, Conejero préc., point 44.

28 HK Danmark, préc, point 88, Conejero, préc., point 48.

29 Voir, en ce sens, arrêt HK Danmark, préc., point 91, Conejero, préc., point 51.

30 Arrêt de la CJUE du 13 juillet 2000, Centrosteel, C-456/98, Recueil de jurisprudence 2000 page I-6007, point 17. 
interprétation par la jurisprudence de la CJUE. Si une interprétation de la règle nationale conforme au droit de l'Union Européenne n'est pas possible, le juge national doit rester libre de laisser inappliquée la disposition nationale qui s'avère contraire au droit de l'U.E.

\section{D - LA RÉFORME DE $2016^{31}$}

Le vide juridique a été comblé avec l'adoption de la loi $89(I) / 2016^{32}$. Avec ce texte, le législateur a allongé la période pendant laquelle il est interdit de donner un préavis de licenciement à un salarié en arrêt-maladie. Dorénavant, l'employeur ne peut pas mettre fin au contrat de travail pour cause d'absence maladie pendant une période maximale de douze (12) mois (période principale de garantie d'emploi), à laquelle s'ajoute une période additionnelle égale à un quart de la durée effective d'absence (période additionnelle de garantie d'emploi), afin de faciliter la réintégration du salarié dans l'entreprise. Par exemple, si le salarié est absent pour cause de maladie pour une période de huit mois, il reste protégé contre le licenciement pendant une période pouvant aller jusqu'à dix (10) mois, (soit les huit mois d'arrêt-maladie et les deux (2) mois supplémentaires). Le salarié peut être protégé pour une période maximale de quinze (15) mois, à savoir douze (12) mois, qui correspondent à la période normale de garantie d'emploi pour cause d'incapacité de travail, et à trois mois additionnels, qui correspondent à la période supplémentaire de garantie d'emploi égale à un quart de la période totale d'absence. La loi assure ainsi que le salarié puisse réintégrer normalement l'entreprise et retrouver ses rythmes habituels.

\section{III - LICENCIEMENT POUR ARRÊT MALADIE ET LICENCIEMENT POUR INSUFFISANCE PROFESSIONNELLE : QUELLE ARTICULATION?}

La nouvelle norme est en concurrence avec le droit de l'employeur de licencier le salarié pour insuffisance professionnelle, puisque l'arrêt maladie peut être assimilé à une inaptitude à exécuter les obligations contractuelles

Le manquement du salarié à exécuter ses fonctions de manière satisfaisante est le cas le plus fréquent de rupture du contrat de travail à l'initiative de l'employeur à Chypre. Le droit de l'employeur de licencier le salarié pour insuffisance professionnelle est l'un des motifs de licenciement licite prévus par la loi 24/67. Entrent dans ce cas les problèmes de santé qui rendent le salarié incapable d'accomplir ses tâches de manière satisfaisante, mais sont expressément exclus les motifs d'incapacité temporaire en raison de maladie, d'accident ou d'accouchement ${ }^{33}$.

Le législateur fait référence à l'existence d'une obligation d'exécuter le travail de manière 'raisonnablement satisfaisante', sans aucune autre indication, et renvoie pour l'interprétation du terme à la jurisprudence. Celle-ci n'exige pas une efficacité maximale ('utmost efficiency') de la part du salarié, mais plutôt une efficacité raisonnable ('reasonable efficiency'), dont l'évaluation est une question de fait selon les circonstances du cas de

31 V. Yannakourou, préc., p. 265.

32 Journal Officiel de la République de Chypre (E.E.) Par. I (I) n 4572/25.7.2016.

33 Art 5 (a) de la L. 24/67. 
l'espèce. Avant de donner un délai de préavis - qui est une condition de toute résiliation du contrat ('notice') à Chypre -, l'employeur doit mettre en garde - si possible par écrit ('warning') - le salarié, pour lui laisser le temps d'améliorer sa performance. L'organisation de réunions avec l'ensemble du personnel ayant les caractéristiques d'assemblées générales, au cours desquelles sont effectuées des insinuations générales et abstraites qui visent tous les salariés, au lieu de plaintes concrètes à l'égard de certains d'entre eux, ne sauraient satisfaire la condition légale de mise en garde. La direction de l'entreprise doit personnaliser ses observations ou ses mises en garde et identifier les salariés qui font l'objet de plaintes ou connaissent des problèmes de performance au travail ${ }^{34}$.

Les cas qui relèvent de ce grief sont, notamment, l'exécution défectueuse des tâches, l'absentéisme continu, les problèmes de santé qui affectent l'aptitude au travail, l'arrivée tardive et l'absence non autorisée du salarié, les performances réduites, l'indolence, l'indifférence, la mise en œuvre de la méthode "go slow», lıobstruction, lıexécution des tâches dans un temps double sans raison apparente, etc. ${ }^{35}$.

Un cas courant d'insuffisance professionnelle dans l'exercice du travail est celui qui résulte des absences fréquentes pour des raisons de santé. Les absences sporadiques pour des raisons de santé entraînent une incapacité de travail temporaire et relèvent des exceptions, c'est-à-dire des cas qui ne justifient pas le licenciement ${ }^{36}$; en revanche, les absences répétées et systématiques, soit de leur propre chef, soit combinées à d'autres paramètres, constituent un motif valable de licenciement, du fait que le salarié manque à l'exécution de son travail de manière raisonnablement satisfaisante ${ }^{37}$. La validité du licenciement dépend à la fois dıune condition de fond et d'une condition procédurale, cette dernière regroupant la méthodologie et la procédure à suivre par l'employeur. La condition essentielle est de savoir si l'employeur a respecté le principe de décision raisonnable. Il est examiné si sa décision est conforme aux réactions raisonnables d'un employeur moyen raisonnable qui aurait agi dans les mêmes circonstances et pour la même raison en disposant des mêmes données. Pour exercer pourtant le droit dont il est muni, l'employeur raisonnable doit observer une procédure appropriée qui assure que les droits du salarié seront pleinement respectés avant que la décision de licenciement ne soit prise ${ }^{38}$. Cette procédure dérive d'une série de principes jurisprudentiels composant le précédent jurisprudentiel en la matière.

La jurisprudence fait peser des obligations procédurales spécifiques sur l'employeur. Ce dernier est obligé d'une part de demander l'avis du salarié lui-même sur la question (consultation), et d'autre part d'obtenir des informations complémentaires par d'autres biais afin d'avoir une opinion complète et fiable sur la maladie du salarié, sa durée et un pronostic sur son évolution. Dans ce cas, il devient parfois nécessaire de consulter le médecin qui suit le malade afin de recueillir le maximum de renseignements.

34 Paraskevi Papachristou v. P.S. loannou Enterprises Ltd (Lenios Beach Hotel) Tribunal de Travail, Application n 81/99 cité par Yannakourou, préc. p. 253.

35 S. Yannakourou, idem.

36 Elles figurent parmi les exceptions prévues à l'article 5 a) de la loi 24/67.

37 Hampis Nikola v. Abattoir Central Kofinou, Appel civil n¹0645 (2001) 1B Arrêts de la Cour Suprême (A.A.D.) 810.

38 Theopistis Andreou v. Louis Hotels Public Company Ltd, Tribunal de Travail, Application n 572/10, arrêt en date du 11.9.2013. 
Le respect d'une procédure pertinente et juste qui tient compte des droits du salarié avant de prendre la décision de licenciement est une des obligations de l'employeur prudent et raisonnable visant à protéger le droit du salarié à son travail ${ }^{39}$. La jurisprudence anglaise à laquelle la jurisprudence chypriote fait souvent référence souligne qu'afin de justifier le licenciement d'un travailleur qui s'absente souvent pour cause de congé de maladie, l'employeur doit procéder à un examen et à une évaluation équitables des absences et des motifs d'absence du salarié avant la prise de la décision de licenciement; à cet effet il peut y avoir recours à tous les moyens appropriés ${ }^{40}$. Le contenu du certificat médical occupe une place importante dans ce processus. Le certificat doit être précis et complet et non pas vague ${ }^{41}$. L'employeur doit tenir compte de la nature de la maladie, la probabilité d'une rechute ou de l'apparition d'une nouvelle maladie, de la durée de chaque période d'absence et la durée des alternances entre périodes d'absence et périodes de présence, ses besoins objectifs, les conséquences des absences du salarié concerné sur les autres salariés, la politique de l'entreprise au sujet des congés maladie et l'impact des absences du salarié malade sur le fonctionnement de l'entreprise ${ }^{42}$.

II résulte que dans certaines conditions la maladie prolongée ou intermittente est assimilée à une insuffisance professionnelle dans l'exécution du travail et peut constituer un motif valable de licenciement selon l'article 5 de la Loi 24/67. En l'occurrence, la protection offerte par la nouvelle loi $86(I) / 2016$ avec l'institution d'une période de garantie d'emploi de quinze (15) mois au maximum, à compter du premier jour de l'apparition de la maladie, ne saurait se plier devant la règle de droit qui autorise le licenciement pour inaptitude professionnelle suite à un arrêt maladie de longue durée. Cette articulation est en cohérence avec l'article 24 de la Charte sociale révisée comme il a été interprété par le Comité européen des droits sociaux (CEDS).

Chypre a ratifié le texte de la Charte sociale européenne révisée le 27/9/2000. L'article 24 de la Charte consacre deux principes juridiques généraux: en premier lieu, le droit de ne pas être licencié sans motif valable (article 24 par a); en second lieu, le droit à une compensation adéquate ou à toute autre réparation en cas de licenciement abusif (article 24 par b). L'Annexe de l'article 24 contient une liste ouverte de motifs qui rendent le licenciement non valable, qui sont liés à la personne du salarié et tirés de la Convention de l'OIT nº 158 et de la Recommandation n 166. Parmi ces motifs figurent des motifs liés à la personne du salarié concernant sa capacité professionnelle ou sa conduite ${ }^{43}$. Ces deux notions n'ont pas été interprétées par le Comité européen des droits sociaux (CEDS)

39 Yiorgos Theodorides v. Louis Hotels Ltd (Ledra Beach Hotel), Tribunal du Travail, Application $n^{\circ}$ 568/06, arrêt en date du 25.9.2009.

40 International Sports Co. Ltd v. Thomson, [1980] Industrial Relations Law Reports (I.R.L.R.) 340.

41 Salamis Tours Ltd v. Christoforos Polykarpou, (2010) 1 A.A.D. 1091.

42 Lynnock v. Cereal Packaging Ltd [1998] Industrial Cases Reports (I.C.R.) 670. V. aussi l'analyse de Polyviou, préc., p. 306.

43 M. Schmidt, "Article 24: The Right to Protection in Cases of Termination of Employment", in N. Bruun, K. Lorcher, I. Schomann et S. Clauwert, The European Social Charter and the employment relation, Hart Publishing, 2016, p. 412 et s., sp.pp. 422-423. 
jusqu'à présent. Deux types d'incapacité professionnelle pourraient être relevés ${ }^{44}$. D'une part, il s'agit du manque d'aptitude et de compétence nécessaire pour exécuter certaines tâches et, de l'autre, il est question de l'incapacité résultant d'une maladie ou d'une lésion. Dans les explications relatives à l'article 24 de la Charte, qui constituent un outil d'interprétation destiné à éclairer les dispositions de ce dernier, il est expressément indiqué ${ }^{5}$ que l'absence temporaire du travail pour cause de maladie n'est pas un motif valable justifiant de mettre fin à la relation du travail à l'initiative de l'employeur. Cependant, la maladie peut devenir constitutive d'un motif valable de licenciement lorsqu'elle dérange gravement la bonne marche de l'entreprise et un véritable remplacement permanent du salarié absent doit être visé. Dans ce cas, un plafond temporel peut être posé à la protection du salarié contre le licenciement, par exemple les premiers six mois suivant une période d'incapacité en raison d'une maladie ${ }^{46}$. II découle de ce qui précède qu'un salarié peut être valablement licencié en raison d'une maladie prolongée qui perturbe gravement le bon fonctionnement de l'entreprise; néanmoins, le licenciement ne peut avoir lieu pendant la période de garantie d'emploi établie par la loi chypriote.

\section{Conclusion}

La loi 24/67 énonce explicitement que l'arrêt maladie temporaire ne constitue pas un motif valable de licenciement. En revanche la loi reste silencieuse sur les effets de la maladie prolongée ou des absences répétées pour des raisons de santé. Cette étude a procédé à une interprétation de la loi chypriote à la lumière de la Charte sociale révisée et des principes jurisprudentiels composant la règle du précédent applicable en la matière. Elle a mis en évidence que les absences de longue durée et celles de caractère systématique sont susceptibles de justifier la rupture du contrat de travail de la part de l'employeur dès lors qu'elles entraînent l'incapacité du salarié de travailler; une telle incapacité professionnelle doit en outre avoir des incidences importantes sur le fonctionnement normal de l'entreprise et nécessiter le remplacement permanent du salarié affecté.

Néanmoins, en droit chypriote l'employeur n'est pas doté d'un droit inconditionnel de licencier un salarié devenu inapte du fait qu'il est longuement ou systématiquement absent de son poste de travail pour cause de maladie. Il doit examiner objectivement si tout autre employeur prudent et raisonnable le ferait à sa place. Afin de conclure, l'employeur doit suivre une procédure raisonnable et juste à la fois, à deux composantes: il doit consulter tant le salarié que d'autres personnes afin d'être sûr de sa décision. La consultation préalable du médecin, après le consentement du salarié concerné, est un élément essentiel de la tentative de l'employeur d'apprendre le véritable état de santé de son salarié afin de mesurer, en tenant compte d'autres critères conjointement, s'il va mettre fin à son contrat ${ }^{47}$. Même lorsque l'employeur juge de façon raisonnable qu'il doit procéder au licenciement pour insuffisance professionnelle du salarié malade, le licenciement ne peut pas avoir lieu au cours de toute la période de garantie d'emploi instituée par la loi ${ }^{48}$.

44 M. Schmidt, préc., p. 423.

45 Article 24, paragraphe 3, point $f$.

46 Conclusions 2005, Norvège, V. Schmidt, préc., p. 423.

47 P Polyviou, préc., p. 317.

48 Loi 89(I)/2016 ayant modifié l'article 9(6) de la loi 24/67. 


\section{L'ARRÊT MALADIE EN DROIT CHYPRIOTE}

L'arrêt du juge du fond, pour sa part, doit être fondé sur tous les faits et les données de l'espèce, et finir par trouver un équilibre entre l'intérêt du salarié à maintenir son poste de travail et l'intérêt de l'employeur de bien faire fonctionner son entreprise. en tous cas, le juge sera amené à adopter des solutions équilibrées, à l'appui de la méthode de la mise en balance des intérêts divergents du salarié et de l'employeur, appliquée au cas par cas et bien ancrée dans sa tradition juridique.

\section{MATINA YANNAKOUROU}

Professeur Assistante en dDroit du travail. Faculté de Droit de l'Université Européenne de Chypre (EUC).

Thèmes de recherche : Discriminations dans le domaine de l'emploi, jurisprudence de la CJUE, économie digitale et relations du travail.

\section{Publications:}

M. Yannakourou (avec D Goulas), "Enforcing Anti-Discrimination Law in Greece: Courts' Resistance and Deficiencies of Civil Litigation against Employment Discrimination" in B. Havelkova and M. Möschel (eds), Anti-Discrimination Law in Civil Law Jurisdictions, Oxford University Press (OUP), 2019, A paraître.

M. Yannakourou (avec G. Tsatiris )(2018), « Les Jeunes et le Droit du Travail en contexte de crise financière » in A Droit Ouvert, Mélanges en I'honneur d'Antoine Lyon-Caen, Paris, Dalloz, 2018, p. 959.

M. Yannakourou, "Trade Union Representativeness and Collective Bargaining: Towards New Rules", Epitheorissis Ergatikou Dikaiou (Labour Law Review), 2018, p. 293 (en grec). 